\title{
Application of Particle Swarm Optimization Based on Beetle Antennae Search Strategy in Wireless Sensor Network Coverage
}

\author{
Dianna Song a) \\ A College of Management Science and Engineering, Shandong Normal University, Shandong 250000, China. \\ a) Corresponding author: 568875103@qq.com
}

\begin{abstract}
Particle swarm optimization has attracted the attention of researchers because of its outstanding performance in optimization problems. The Beetle Antennae search algorithm(BAS) is a newly proposed simple and efficient search algorithm. This paper combines it with the particle swarm optimization algorithm, applies the individual search method to the group search, validates its validity through the test function, and applies Wireless sensor network coverage problem.
\end{abstract}

Key words: Particle Swarm Optimization; Beetle Antennae Search Algorithm; Wireless Sensor Network Coverage.

\section{INTRODUCTION}

Particle Swarm Optimization (PSO) is a typical intelligent optimization algorithm. Since it was published in the form of a paper, it has attracted the attention of a large number of researchers. It has been mentioned and cited in various academic conferences and has formed a relatively independent discipline. Its papers are also included in a large number of databases and are used in intelligent optimization, neural networks, data mining, power transmission, vehicle distribution, signal processing, smart machines, control engineering, biopharmaceuticals, shop scheduling, information dissemination, network transmission, etc. A large number of areas that need to be optimized make it one of the most widely used intelligent optimization algorithms. In theory, this paper proposes a particle swarm optimization algorithm based on the Beetle Antenna Search Strategy Particle Swarm Optimization. In the application, the improved algorithm will be applied to the wireless sensor network coverage problem and combined with appropriate Examples to prove the feasibility and effectiveness of the improved algorithm. The improved algorithm is applied to the wireless sensor network coverage problem to achieve the optimization of wireless sensor network coverage and provide scientific basis for the optimization strategy of wireless sensor network coverage.

\section{THE MATHEMATICAL MODEL OF WIRELESS SENSOR NETWORK COVERAGE}

\section{Problem Analysis}

The coverage of the sensor node is a fixed radius circle with its own center dots. To determine whether a position is within the coverage of the sensor node, it is necessary to calculate whether it is within the circle of the sensor node by calculating the distance. The location in the region is continuous. In order to simplify the coverage problem in the region, the measured area is discretized into $\mathrm{m} \times \mathrm{n}$ pixels. Assuming that there are $\mathrm{x}$ pixels can be covered by the WSN network, the coverage is $\mathrm{x} /(\mathrm{m} \times \mathrm{n})$. 
All sensor nodes in the WSN network have the same perceived radius and communication radius in the WSN network. If the WSN network can realize the full coverage of the sensing area when the communication radius is greater than or equal to twice, then all sensor nodes must connect.

\section{Coverage Model Description}

$\mathrm{C}^{\prime}$ is a subset of $\mathrm{C}$, and $\mathrm{A}\left(\mathrm{C}^{\prime}\right)$ expresses the cover area of node set $\mathrm{C}^{\prime}$, and regional coverage could be defined as $\mathrm{PCOV}=\mathrm{A}\left(\mathrm{C}^{\prime}\right) / \mathrm{A}(\mathrm{C})$. In practice, directly calculating the value of $\mathrm{A}(\mathrm{C})$ is complex; to simplify the calculation, detection area $A$ will grid into $M^{*} N$ points, the coordinates of the lattice are (x,y), the distance from grid point to its sensor is defined as $d(c i, p)=\sqrt{(x-x i) 2+(y-y i) 2}$. Define the probability of grid points covered by sensor nodes as

$$
\mathrm{p}(\mathrm{ci}, \mathrm{t})=\left\{\begin{array}{lr}
1 & d(c i, t k) \leq r \\
0 & \text { else }
\end{array}\right.
$$

When grid point $(\mathrm{xk}, \mathrm{yk})$ covered by more than one sensor, mark it as covered state, and the probability of grid points been covered is defined as

$$
\mathrm{p}(\mathrm{ci}, \mathrm{t})=1-\prod_{t i \in T}[1-p(c i, t k)]
$$

so, the coverage is defined as

$$
\operatorname{PCOV}=\frac{\sum_{c \in M \times N} p(c, t)}{M \times N}
$$

\section{THE GROUP INTELLIGENT OPTIMIZATION STRATEGY BASED ON BEETLE ANTENNAE SEARCH}

\section{Basic Principle of Beetle Antennae Search Algorithm}

The Beetle Antennae Search-BAS, also called the Beetle Search, is an efficient intelligent optimization algorithm proposed in 2017. Similar to the intelligent optimization algorithms such as genetic algorithm, particle swarm optimization, and simulated annealing, Antennae must search without knowing the specific form of the function, and without gradient information, can achieve efficient optimization. Compared to particle swarm optimization, Antennae must search only one individual, namely a long-horned cow, and the computational load is greatly reduced. Antennae must search is an algorithm developed in response to the inspiration of the antennae foraging principle. When the cows were feeding on the day, antennae did not know where the food was, but grazing based on the smell of food. Antennae has two long tentacles. If the odor intensity received by the left antennae is greater than the right one, then antennae will fly to the left, otherwise it will fly to the right. Based on this simple principle, antennae can effectively find food.

Antennae must search for our inspiration: the smell of food is equivalent to a function, this function is different in each point of the three-dimensional space, antennae two must be able to collect the odor value of two points near their own, antennae purpose is Find the point with the largest global odor value. By following the behavior of antennae, we can efficiently perform function optimization. Antennae moves in three-dimensional space, and antennae must search for it to be valid for any dimension function. Therefore, antennae must search is a generalization of the biological behavior of antennae in any dimension space.

We use the following simplified model assumptions to describe the antennae search process:

1. Both sides of antennae must be located on both sides of the centroid.

2. The ratio of the distance $\mathrm{d} 0$ between the step and the two whiskers is a fixed constant, ie $\mathrm{step}=\mathrm{c} * \mathrm{~d} 0$, where $\mathrm{c}$ is a constant. That is, big antennae (two must be long distances) take a big step and small antennae takes a small step.

3. After antennae flies to the next step, the head is random. 


\section{Intelligent Optimization Based on BAS Algorithm}

The Particle Swarm Optimization (PSO) algorithm is an algorithm based on the social behavior of a flock of birds that was developed by Kennedy and Eberhart. The motion of the particles is interpreted as birds flying. The particles move in the searching space according to their former speed, their experience, and the experience of their surrounding neighbors. The dth dimension ith particle (xid) represents a potential solution of the optimization. The dimension of the particle represents the number of variables that need to be optimized.

In the process of the PSO algorithm, the position and the velocity of each particle evolve as follows:

$$
\begin{aligned}
\operatorname{Vij}(t+1)= & w V i j(i)+c 1 \times r 1 \times(\text { Pbest-Xij })+c 2 \times r 2 \times(\text { Gbest-Xij) } \\
& X i j(t+1)=\operatorname{Xij}(t)+\operatorname{Vij}(t+1)
\end{aligned}
$$

where $\mathrm{w}$ is an inertia factor which ensures that the direction of motion of a particle is affected by its former velocity. It must be smaller than one and usually linearly decreases from 0.9 to 0.4 with respect to the time t. $\mathrm{r} 1$ and $\mathrm{r} 2$ are independent random number from 0 to 1 . Pbest represents the best position ever found for the i-th particle and Gbest represents the global best position. $\mathrm{c} 1$ and $\mathrm{c} 2$ are respectively the cognitive factor and social factor which control the motion of the particle to its personal best position and global best position. The position of the particle is renewed by the velocity. The PSO algorithm will stop when the maximum number of iterations is met.

The improved algorithm steps are as follows:

Step 1: In the j-dimensional problem space, the initial position and velocity of i particles are randomly generated, and initialization parameters are used.

Step 2: Use the fitness function to calculate the fitness of each particle.

Step 3: Randomly generate a direction dir for each particle, determine the left and right coordinates $\mathrm{xl}$ and $\mathrm{xr}$, compare the values of $f(x l)$ and $f(x r)$, update the particle position according to equation (6), calculate the fitness value and Pbest for comparison, if the current searched fitness value is better than Pbest, Pbest is updated with the new fitness value and location of the particle.

$$
\mathrm{x}=\mathrm{x} \text {-step*dir*sign }(\mathrm{f}(\mathrm{xl})-\mathrm{f}(\mathrm{xr}))
$$

Step 4: Compare the current searched fitness value with the Gbest of all the particles in the population. If the current searched fitness value is better than Gbest, update the Gbest value and position.

Step 5: Change the speed and position of the particles according to Equation (4)(5).

Step 6: Go back to Step 2 and repeat until you reach the maximum number of iterations or reach the desired fitness value.

\section{Algorithm Simulation and Analysis}

In order to verify the effectiveness of the proposed algorithm, the algorithm is compared with the standard particle swarm optimization to solve four test functions. In order to facilitate the programming and display the results of the algorithm, all the experiments in this paper are running in the version of matlab7.0, using MATLAB language, the same HP machine running under the same environment, the machine is configured for Win7 operating system, The processor is Intel i3, $2.10 \mathrm{GHZ}$, video card size is $1 \mathrm{G}$, memory size is $2 \mathrm{G}$.

The setting of the parameters in the algorithm has a great influence on the effect of the algorithm. By consulting a large amount of literature and conducting simple experimental tests, the parameters in the algorithm are set as follows: the size of the population ps $=30$; in order to make the algorithm have an expanded search range in the initial stage, The inertia factor is updated with the following formula $\omega=\omega_{\max }-\left(\frac{\mathrm{k} *\left(\omega_{\max }-\omega_{\min }\right)}{\operatorname{Maxnum}}\right), \omega_{\max }$ set to 0.9 and $\omega_{\min }$ set to 0.4 ; the parameters $C_{1}, C_{2}$ are set to the same number $C_{1}=C_{2}=2$; the iterations of PSO are 100 , $300,500,1000$, and 2000. In order to ensure the fairness of the result, each algorithm runs 100 times independently. 
TABLE 1. Algorithm Comparing

\begin{tabular}{|c|c|c|c|c|c|}
\hline Iterations & Algorithm & Sphere & Griewank & Rastrigin & Rosebrock \\
\hline \multirow{2}{*}{100} & BASPSO & 63.98 & 0.89 & 264 & 67832 \\
\hline & PSO & 236.8 & 2.9 & 716 & 69928 \\
\hline \multirow{2}{*}{300} & BASPSO & 24.71 & 0.63 & 213 & 23692 \\
\hline & PSO & 49.9 & 1.4 & 388 & 68954 \\
\hline \multirow{2}{*}{500} & BASPSO & 14.15 & 0.54 & 178 & 13989 \\
\hline & PSO & 23.05 & 1.2 & 315 & 67924 \\
\hline \multirow{2}{*}{1000} & BASPSO & 2.47 & 0.35 & 126 & 4057 \\
\hline & PSO & 6.13 & 1.04 & 248 & 67194 \\
\hline \multirow{2}{*}{2000} & BASPSO & 0.32 & 0.12 & 103 & 1274 \\
\hline & PSO & 1.67 & 0.84 & 193 & 67682 \\
\hline
\end{tabular}

The comparison results are shown in Table 1. It can be seen from the experiment that as the number of iterations increases, the running result of the algorithm gradually approaches the optimal value. In the optimization process, the BASPSO converges faster and the accuracy of the final result is better.

The improved algorithm is applied to wireless sensor network coverage problem and compared with the standard particle swarm algorithm. It is assumed that the area of the wireless sensor network monitoring area is a square area of $50 \mathrm{~m} \times 50 \mathrm{~m}$. The sensor node has a sensing radius of $5 \mathrm{~m}$ and the number of sensors is 40 . Simulation experiments were performed on the MATLAB platform. The comparison results are as follows.

TABLE 2. Wireless Sensor Network Coverage Comparison.

\begin{tabular}{ccc}
\hline Algorithm & PSO & BASPSO \\
\hline Mean & 0.86479 & 0.90198 \\
Optimum & 0.93388 & 0.95041 \\
\hline
\end{tabular}

It can be seen that in practically solving the problem of wireless sensor network coverage, the improved algorithm is obviously better than the standard particle swarm algorithm. Wireless sensor network coverage simulation experiment as shown below.

\section{ACKNOWLEDGMENTS}

This work was supported by the Natural Science Foundation of China (No.61502283 and No.61472231).

\section{REFERENCES}

1. Slipinski A, Escalona H. Australian Longhorn Beetles (Coleoptera: Cerambycidae) Volume 1[M]. 2013.

2. Zhang Y, Li S. PSA: A novel optimization algorithm based on survival rules of porcellio scaber[J]. 2017.

3. Jiang X, Li S. BAS: Beetle Antennae Search Algorithm for Optimization Problems[J]. 2017.

4. ZHANG W, MA D, WEI J, et al. A parameter selection strategy for particle swarm optimization based on particle positions[J]. Expert Systems with Applications,2014,41(7):3576-3584.

5. KUNDU R, DAS S, MUKHERJEE R, et al. An improved particle swarm optimizer with difference mean based perturbation[J]. Neurocomputing,2014,129(10):315-333.

6. Howard A, Matarić MJ, Sukhatme GS. Mobile sensor network deployment using potential field: A distributed scalable solution to the area coverage problem. In: Proc. of the 6th Int'l Sympon Distributed Autonomous Robotics Systems (DARS 2002). 2002.299-308.

7. Ji Zhen, Liao Huilian, Wu Qinghua. Particle Swarm Optimization Algorithm and Its Application[M]. Beijing: Science Press, 2009.

8. Singh A, Sharma T P. A survey on area coverage in wireless sensor networks[C]//International Conference on Control, Instrumentation, Communication and Computational Technologies,2014:829-836.

9. Mostafaei H, Meybodi M R. Maximizing Lifetime of Target Coverage in Wireless Sensor Networks Using Learning Automata[J]. Wireless Personal Communications,2013,71(2):1461-1477. 